\title{
Comparison of Multilevel Wavelet Packet Entropy using Various Entropy Measurement for Lung Sound Classification
}

\author{
Achmad Rizal $^{1}$, Risanuri Hidayat ${ }^{2}$, Hanung Adi Nugroho ${ }^{3}$ \\ School of Electrical Engineering, Telkom University, Bandung, Indonesia ${ }^{1}$ \\ Department of Electrical Engineering \& Information Technology, Universitas Gadjah Mada, Yogyakarta, Indonesia ${ }^{1,2,3}$
}

\begin{abstract}
Wavelet Entropy (WE) is one of the entropy measurement methods by means of the discrete wavelet transform (DWT) subband. Some of the developments of WE are wavelet packet entropy (WPE), wavelet time entropy. WPE has several variations such as the Shannon entropy calculation on each subband of WPD that produces 2N entropy or WPE, which yields an entropy value. One of the WPE improvements is multilevel wavelet packet entropy (MWPE), which yields entropy value as much as $\mathbf{N}$ decomposition level. In a previous research, MWPE was calculated using Shannon method; hence, in this research MWPE calculation was done using Renyi and Tsallis method. The results showed that MWPE using Shannon calculation could yield the highest accuracy of $97.98 \%$ for $N=4$ decomposition level. On the other hand, MWPE using Renyi entropy yielded the highest accuracy of $93.94 \%$ and the one using Tsallis entropy yielded $57.58 \%$ accuracy. Here, the test was performed on five lung sound data classes using multilayer perceptron as the classifier.
\end{abstract}

Keywords-Wavelet packet entropy; lung sound; Shannon entropy; Renyi entropy; Tsallis entropy

\section{INTRODUCTION}

Abnormalities that occur in the respiratory system can be observed from the sound generated during the respiratory process. This breathing sound commonly is heard by a doctor using a stethoscope, also known as auscultation. The respiratory or pulmonary sound analysis is one of the most interesting research topics in the field of medical signal processing. Various methods have been developed for the extraction of pulmonary sound features for automatic classification. One of the most commonly used methods is the entropy analysis. Entropy is a measure of signal or system irregularity. It is frequently used to measure signal complexity as in biological signals.

Various entropy calculation methods have been used in lung sound analysis in which Sample entropy was used as a feature for detecting pulmonary sound status using morphological complexities [1]. Meanwhile, Tsallis entropy was used for lung sound analysis in [2] and [3]. Multiscale entropy was reported to be better in distinguishing lung sounds in alveolitis patients rather than spectral or statistical methods [4]. Another entropy measurement method is the wavelet entropy (WE). It uses Shannon entropy calculations on the subband of discrete wavelet transform (DWT) [5]. The improvement of the wavelet entropy is the wavelet packet entropy (WPE) that uses the wavelet packet decomposition (WPD) subband [6]. WE produced low accuracy when used as a feature for pulmonary sound classification as reported in [7]. In five classes of pulmonary data, WE only resulted in an accuracy of $43 \%$. For that reason, the development of WE method is needed to improve the accuracy of pulmonary sound classification.

Previous research has proposed a multilevel wavelet packet entropy (MWPE) method for pulmonary sound feature extraction [8]. Entropy was calculated on the subband of WPD at several levels using the Shannon entropy method. In addition to the Shannon method, there are other several methods of calculating entropy such as Renyi entropy and Tsallis entropy. Renyi entropy is a common form of Shannon entropy [9]; while Tsallis entropy is a generalization form of entropy with the generalization parameter of $\mathrm{q}$ [10]. In this study, MWPE was calculated using Renyi entropy and Tsallis entropy to observe the resulted accuracy. The results obtained was then compared with the MWPE resulted in previous studies using five classes of lung sound data.

This paper is presented as follows. Section 2 describes some previous studies using wavelet entropy and wavelet packet entropy. Section 3 describes the detailed methods used in this study including data, wavelet decomposition, and classifier. Results and discussion are presented in Section 4 and the conclusions and prospects for future research are presented in Section 5.

\section{RELATED WORKS}

Wavelet entropy (WE) is widely used for complex signal analysis such as for biological signals. It is entropy calculation using subband of DWT. In [5], WE was used for brain signals analysis in short durations. Compared to spectral entropy (SE), WE was found better to detect non-stationary signals. It was also used for a ventricular beat suppression analysis in the cases of atrial fibrillation [11]. A number of differences in WE values showed some different levels of suppression in the ventricular beat. If WE was calculated on the subband of the DWT, then some researchers used subband results from WPD. Entropy in the subband of WPD results were used for analysis of murmurs in heart sound in [12]. Not all subbands were used for entropy calculation but they could be calculated based on the frequency range, noise frequency, and energy threshold [12]. 
Another variation of WE was the different entropy calculations on wavelet subband. Sample entropy on DWT subband used for EEG signal analysis was presented in [13]. Cen and Li used the Tsallis wavelet entropy for power signal analysis [14]. Normalized Shannon wavelet entropy, meanwhile, was calculated on wavelet coefficient for epileptic EEG analysis [15].

Another method based on wavelet entropy is the wavelet packet entropy (WPE). Some variations of WPE have been proposed by several researchers. In [6], entropy was calculated using crest energy in each subband of WPD results. Meanwhile, Shannon method was used in WPD subband for bearing inspection in [16]. The number of generated features was $2^{\mathrm{N}}$, where $\mathrm{N}$ refers to the signal decomposition level. In another study, multilevel wavelet packet entropy (MWPE) was proposed for lung sound analysis [8]. If in [16], WPE was produced by calculating the Shannon entropy on each WPD subband, then in [8], WPE was generated from Shannon entropy calculations from the subband relative energy such as WE calculation in [5]. So, each decomposition level would produce an entropy value. Since WPE was calculated on multilevel, for $\mathrm{N}$ level decomposition it will produce $\mathrm{N}$ entropy values as the signal feature. The experiments reported $97.98 \%$ accuracy using Db8 at the level of decomposition $\mathrm{N}=4$ [8]. The results were obtained for five classes of lung sound data.

In previous research, MWPE used Shannon entropy calculation to compute entropy on WPD subband. In this study, MWPE was tested using Renyi entropy (RE) and Tsallis entropy (TE). Renyi entropy is a common form of the entropy equation [9]. Meanwhile, Tsallis entropy, commonly called as non-extensive entropy, is often used for non-additive signal analysis [17]. A comparison among ShEN, RE, and TE for MWPE calculation is expected to be a recommendation of the selection of entropy calculation methods on MWPE for biological signal analysis, especially for lung sound analysis.

\section{MATERIAL AND METHODS}

Fig. 1 shows a block diagram of the process conducted in this paper. First, the preprocessing of the pulmonary sound signal was done for amplitude normalization and to uniform the mean of the signal. WPD was then performed from level 1 to level N. At each level of decomposition it performed WPE calculations using the Shannon, Renyi, and Tsallis method. In the next stage, the classification was done using MLP and Nfold cross-validation. A further explanation is provided in the following subsections.

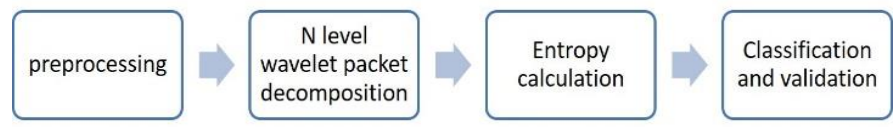

Fig. 1. The Block Diagram of Lung Sound Classification using MWPE.

\section{A. Lung Sound Data}

In this study, we used lung sound recording data obtained from several sources [18][19]. The same data was used in previous studies [8][20]. Each pulmonary sound data consisted of single breathing cycle, inspiration, and expiration. Using a sampling frequency of $8000 \mathrm{~Hz}$, the length of one data was then ranged from 20000-30000 samples. The data consisted of five classes of normal bronchial data representing normal lung sounds, wheeze, stridor, crackle, and plural rub representing pathological lung sound [21]. In the data normalization process was carried out as follows. Zero-mean was done as in Equation (1).

$y(n)=x(n)-\frac{1}{N} \sum_{i}^{N} x(i)$

with $x(n)$ is the input signal and $y(n)$ is the output signal, which has mean $=0$. Furthermore, the normalization of amplitude was presented as in Equation (2) in order to obtain a signal in the range of -1 to +1 .

$y(n)=\frac{x(n)}{\max |x|}$

In the next step, we did wavelet decomposition to calculate the WPD as in the following section.

\section{B. Wavelet Packet Entropy}

Wavelet packet decomposition (WPD) on signal $\mathrm{S}(\mathrm{t})$ was defined as in equation (3).

$d_{j, n}(k)=2^{\frac{j}{2}} \int_{-\infty}^{+\infty} S(t) \psi_{n}\left(2^{-j} t-k\right) d t$,

$0 \leq n \leq 2^{N}-1$

with $\mathrm{S}(\mathrm{t})$ is an original signal, $\mathrm{j}$ is the scale, $\mathrm{n}$ and $\mathrm{k}$ are the band and surge parameter respectively. From Equation (3) we could calculate the energy of each subband as in Equation (4).

$$
E_{j, n}=\sum_{k}\left|d_{j, n}(k)\right|^{2}
$$

where $\mathrm{j}, \mathrm{n}, \mathrm{k}$ represent the scale, band, and surge parameter, respectively. The total energy of WPD is:

$E_{\text {tot }}=\sum_{n} E_{j, n}$

Relative energy for each subband in scale $\mathrm{j}$ can be expressed as:

$p_{j, n}=\frac{E_{j, n}}{E_{t o t}}$

Wavelet packet entropy (WPE) is expressed as:

$W P E_{N}=-\sum p_{j, n} \ln p_{j, n}$

The $\mathrm{N}$ notation is used to denote the level of decomposition used in WPD. 
In the previous study, we used one WPE value as a feature for signal analysis. In this paper, it is proposed to use N WPE value for the feature extraction of the pulmonary sound signal to improve accuracy in pulmonary sound classification. The characteristics used in this study are as in (8).

$M W P E=\left[W P E_{1}, W P E_{2}, \ldots, W P E_{N}\right]$

Equation (7) uses the Shannon method to calculate WPE. This equation can be modified using Renyi entropy or Tsallis entropy. The calculation of Renyi entropy for WPE can be expressed by Equation (9).

$W P E r_{N}=\frac{1}{1-q} \log _{2}\left(\sum p_{j, n}^{q}\right), q \neq 1$

where $r$ is the notation in which WPE was calculated using Renyi entropy (RE), $\mathrm{N}$ is the decomposition level, and $\mathrm{q}$ is the order. Practically, we used the order of $\mathrm{RE} q=2$.

Meanwhile, WPE with Tsallis Entropy method can be calculated using equation (10)

$W P E t_{N}=\frac{1-\sum p_{j, n}^{q}}{q-1}$

Where, $\mathrm{t}$ is the notation in which WPE was calculated using the Tsallis entropy (TE) method, $\mathrm{N}$ is the decomposition level, and $q$ is the order. Here, we used TE order $q=2$.

MWPE would be calculated at the decomposition level $\mathrm{N}=$ 1-7. Based on the results of previous research, a higher level of decomposition of $\mathrm{N}>7$ will not improve accuracy [8]. The mother wavelets tested were Haar, Db2, Db8, Bior1.5, and Bior2.8 as used in [8].

\section{Classifier and Validation}

In this study, we used multilayer perceptron (MLP) as the classifier and $\mathrm{N}$-fold cross-validation (Nfold $\mathrm{CV}$ ) for validation. MLP and Nfold CV were selected as the classifiers in which the results obtained would be compared with previous studies that used the same classifier and validation. MLP was chosen because of its simple architecture and its ability to solve non-linear problems. MLP does not require a large amount of training data to learn [22]. MLP consists of the input layer, hidden layer, and the output layer. The number of features determine the number of node in input layer; while the number of nodes in the output layer corresponds to the number of data classes. Meanwhile, the number of hidden layers and the number of nodes in the hidden layer were determined by trial and error. Basic configuration of MLP is displayed in Fig. 2. For MLP parameters we used learning rate 0.3 , momentum 0.2 , epochs 500, and sigmoid as activation function. We did not choose the best parameter for MLP because we wanted to focus on the effect of MWPE as features. In this study, we used 3 fold CV; the overall data was divided into three datasets with one dataset used as test data and two datasets used as training data. Testing was done three times so that all dataset ever used once as test data. We chose 3FCV because the least amount of data in one class is 18 so that at least each data set will consist of six data.

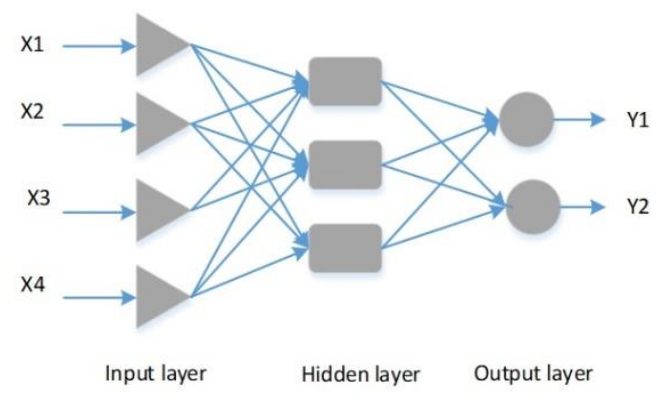

Fig. 2. MLP Configuration.

\section{RESULTS AND DISCUSSION}

The result of wavelet packet decomposition up to level 2 for wheeze sound can be seen in Fig. 3. It appears the information of the signal concentrated at low frequency. Subband AA2 occupied the band $0-1000 \mathrm{~Hz}$ indicating that the most of the lung sound energies lied in the frequency $<1000$ $\mathrm{Hz}$. Thus, a decomposition level $\mathrm{N}>2$ was required to view information from the lung sound. In this research, we used the level of decomposition $\mathrm{N}=7$ so that the subband can be as wide as $31.75 \mathrm{~Hz}$.

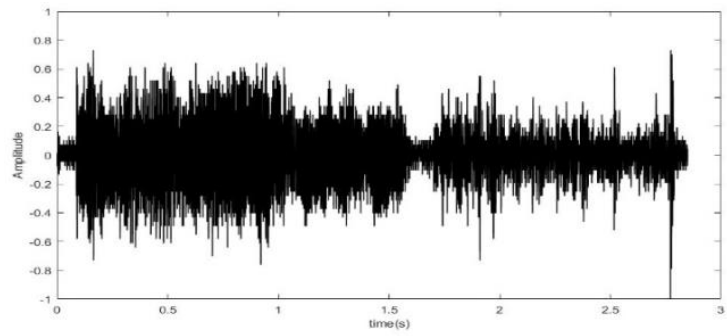

(a) (b)

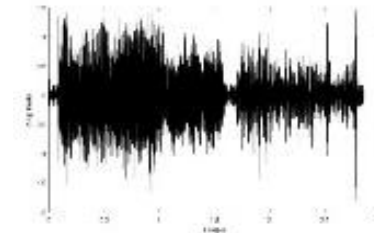

(d)

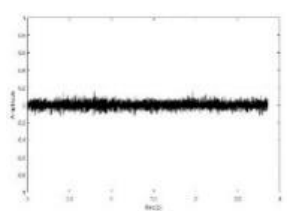

(f)

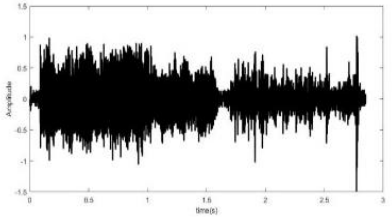

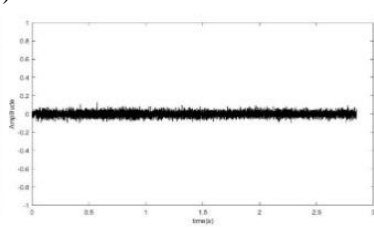

(c)

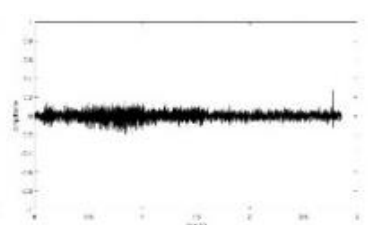

(e)

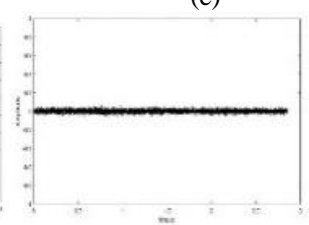

(g)
Fig. 3. Wheeze Sound and its Wavelet Packet Decomposition Result (a) Wheeze Sound (b) Subband A1 (c) Subband D1 (d) Subband AA2 (e) Subband AD2 (f) Subband DA2 (g) Subband DD2. 
Fig. 4 shows the MWPE results for each entropy calculation. Fig. 4(a) shows MWPE using Shannon method. Shannon method generated WPE values that increased along with increasing levels of decomposition. This was because the energy in each subband spread more evenly, especially for the frequencies below $1000 \mathrm{~Hz}$. The WPE value of each decomposition level for each class was also relatively far apart, so there was a difference between each type of lung noise. Stridor produced the highest WPE value, while the pleural rub produced the lowest one. Stridor had a more evenly distributed signal spectrum $<1000 \mathrm{~Hz}$ while the pleural rub had a spectrum that tended to be concentrated at one frequency. Comparison between Stridor and pleural rub can be seen in Fig. 5 and 6.

Fig. 4(b) shows MWPEr in five classes of lung sound data. Crackle and stridor had the highest value, and relative coincide while wheeze, normal, and pleural rub resulted in lower values. The MWPEr value tended to increase as the $\mathrm{N}$ value rose but not so high. The minus sign was generated from the factor (1q) with $q=2$. As shown in Fig. 3, some MWPEr values coincided at some $\mathrm{N}$ levels, causing the possibility of relatively high classification errors.

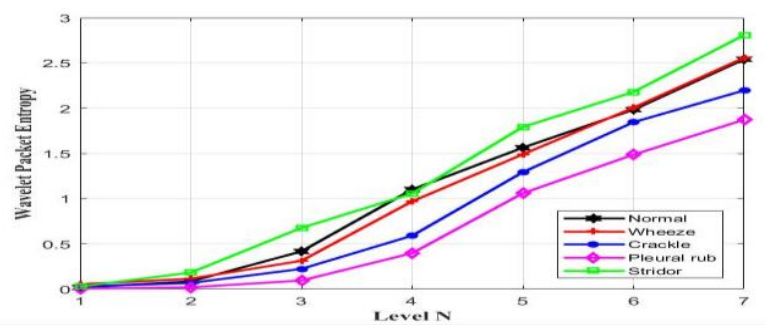

(a)

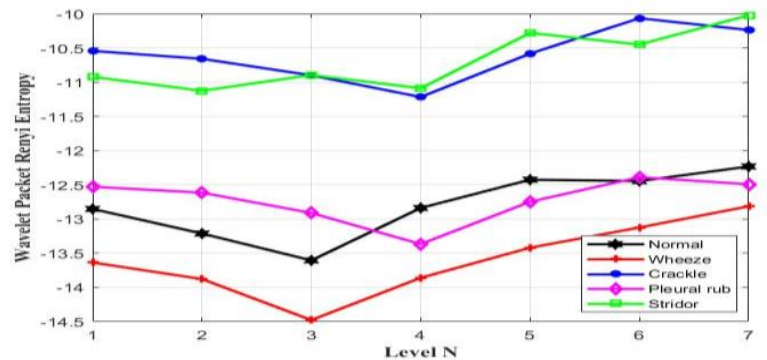

(b)

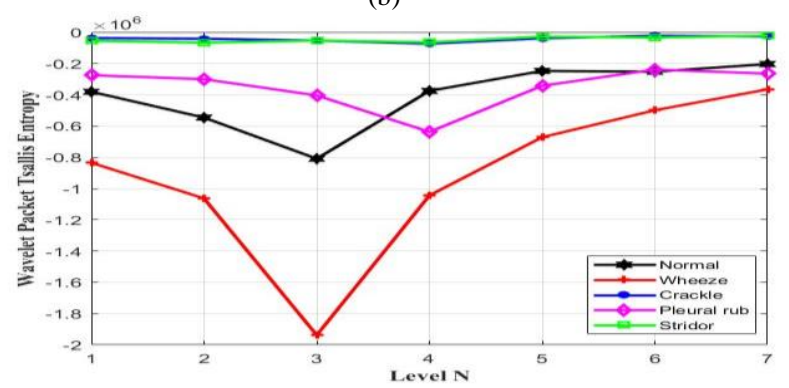

(c)

Fig. 4. Wavelet Packet Entropy using (a) Shannon Entropy for N Level Decomposition (b) Renyi Entropy for N Level Decomposition (c) Tsallis Entropy for N Level Decomposition.
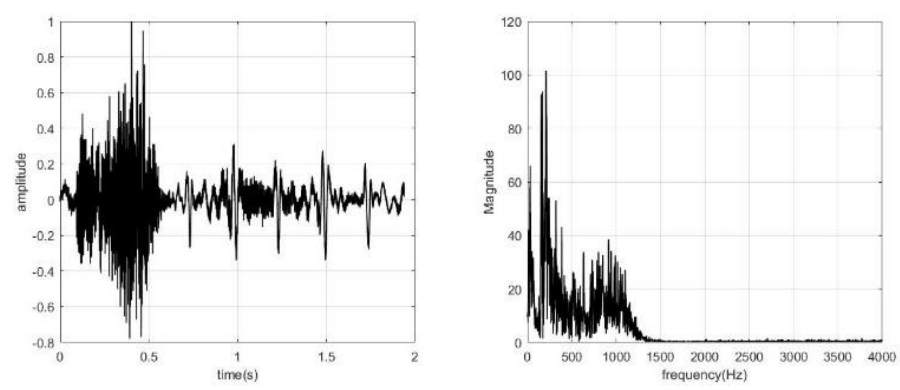

Fig. 5. Stridor and Frequency Spectrum.
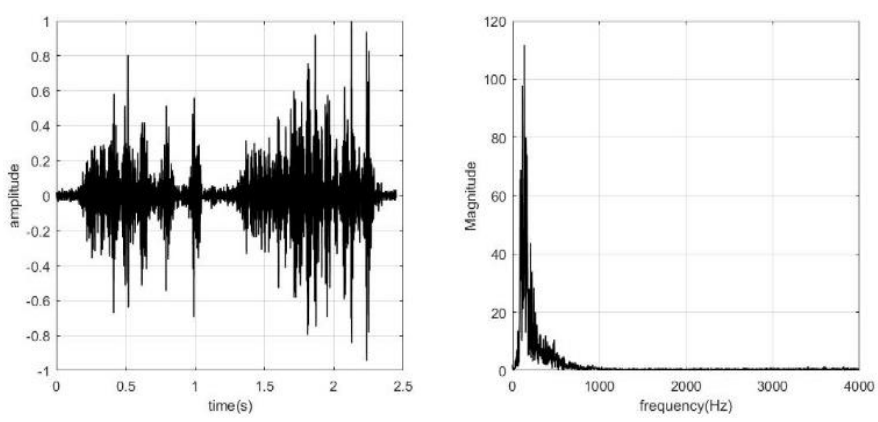

Fig. 6. Pleural rub and Frequency Spectrum.

Fig. 4(c) shows MWPEt in five classes of lung sound data. The results showed the same pattern as MWPEr where crackle and stridor had relatively close values while normal, wheeze, and pleural rub had lower values with the same pattern. MWPEt had the same pattern as MWPEr because it had the same form of the equation and was calculated in a same order. MWPEt had a higher magnitude compared to MWPEr.

The accuracy of lung sound classification using MWPE, MWPEr, and MPWEt is presented in Table 1-Table 3. In Table 1 , the highest accuracy using MWPE was $97.98 \%$ using Db8 with decomposition level $\mathrm{N}=4$ [8]. The $97.98 \%$ accuracy was also achieved when $\mathrm{N}=5-7$ but $\mathrm{N}=4$ was taken as the best parameter for producing the fewest features. It is generally seen that the accuracy increases when the $\mathrm{N}$ value rises and then stable over $\mathrm{N}=4$. At $\mathrm{N}=4,16$ subbands (24) would be formed with a width of $250 \mathrm{~Hz}$ on each. Because of the most significant information lying in the frequency $<1000 \mathrm{~Hz}$, then four subbands of $250 \mathrm{~Hz}$ were sufficient as a differentiator between data classes. It can be seen in Fig. 2 that for $\mathrm{N}=4$ there is a considerable difference between the data classes.

MWPEr produced the highest accuracy of $93.94 \%$ using Bior2.8 and the decomposition level $\mathrm{N}=4$. For higher $\mathrm{N}$, the accuracy value was unchanged. This result was similar with a result on MWPE where a higher level of decomposition did not produce a higher accuracy. MWPEt only produced the highest accuracy of $57.58 \%$ for Bior1.5 with $\mathrm{N}=3$. The higher decomposition rate did not improve the accuracy. This low accuracy was due to very high WPEt values and tended to spread. 
TABLE I. The ACCURACY OF MWPE (\%) For VARIOUS MOTHER WAVELETS AND DECOMPOSITION LEVEL N [8]

\begin{tabular}{|l|l|l|l|l|l|l|l|}
\hline \multirow{2}{*}{ Mother wavelet } & \multicolumn{2}{|l|}{ Multilevel } & $\mathbf{N}=\mathbf{2}$ & $\mathbf{N = 3}$ & $\mathbf{N = 4}$ & $\mathbf{N}=\mathbf{5}$ & $\mathbf{N}=\mathbf{6}$ \\
\cline { 2 - 8 } & $\mathbf{N = 1}$ & 80.81 & 88.89 & 91.92 & 90.91 & 92.93 & 92.93 \\
\hline Haar & 63.64 & 86.87 & 95.96 & 95.96 & 97.98 & 93.94 & 96.97 \\
\hline Db2 & 69.7 & 77.78 & 91.92 & 97.98 & 97.98 & 97.98 & 97.98 \\
\hline Db8 & 53.54 & 81.82 & 88.89 & 91.92 & 93.94 & 90.91 & 93.94 \\
\hline Bior1.5 & 66.67 & 82.83 & 92.93 & 96.97 & 96.97 & 96.97 & 96.97 \\
\hline
\end{tabular}

TABLE II. THE ACCURACY OF MWPER (\%) FOR VARIOUS MOTHER WAVELETS AND DECOMPOSITION LEVEL N

\begin{tabular}{|c|c|c|c|c|c|c|c|}
\hline \multirow{2}{*}{ Mother wavelet } & \multicolumn{7}{|c|}{ Multilevel } \\
\hline & $\mathbf{N}=\mathbf{1}$ & $\mathbf{N}=\mathbf{2}$ & $\mathbf{N}=\mathbf{3}$ & $\mathbf{N}=4$ & $\mathbf{N}=\mathbf{5}$ & $N=6$ & $\mathrm{~N}=7$ \\
\hline Haar & 64.65 & 59.6 & 66.67 & 64.65 & 64.65 & 66.67 & 67.68 \\
\hline $\mathrm{Db} 2$ & 65.66 & 63.64 & 71.72 & 75.76 & 70.71 & 72.73 & 76.77 \\
\hline Db8 & 65.66 & 63.64 & 80.81 & 82.83 & 80.81 & 80.81 & 86.87 \\
\hline Bior1.5 & 65.66 & 63.64 & 81.82 & 87.88 & 86.87 & 93.94 & 90.91 \\
\hline Bior2.8 & 66.67 & 65.66 & 89.9 & 93.94 & 93.94 & 93.94 & 93.94 \\
\hline
\end{tabular}

TABLE III. The ACCURACY OF MWPET (\%) FOR VARIOUS Mother WAVELETS AND DECOMPOSITION LEVEL N

\begin{tabular}{|c|c|c|c|c|c|c|c|}
\hline \multirow{2}{*}{ Mother wavelet } & \multicolumn{7}{|c|}{ Multilevel } \\
\hline & $\mathbf{N}=\mathbf{1}$ & $\mathbf{N}=\mathbf{2}$ & $\mathbf{N}=\mathbf{3}$ & $N=4$ & $\mathbf{N}=\mathbf{5}$ & $N=6$ & $N=7$ \\
\hline Haar & 55.56 & 56.57 & 53.53 & 53.53 & 53.53 & 52.52 & 53.53 \\
\hline $\mathrm{Db} 2$ & 55.56 & 56.57 & 53.53 & 55.56 & 53.53 & 48.48 & 50.5 \\
\hline $\mathrm{Db} 8$ & 55.56 & 57.58 & 53.53 & 55.56 & 54.54 & 48.48 & 52.52 \\
\hline Bior1.5 & 55.56 & 56.57 & 57.58 & 56.57 & 54.54 & 49.49 & 54.55 \\
\hline Bior2.8 & 54.55 & 53.54 & 51.51 & 53.53 & 53.53 & 51.51 & 47.47 \\
\hline
\end{tabular}

From the simulation results obtained that MWPE using Shannon entropy yielded the highest accuracy of $97.98 \%$ using Db8 and $\mathrm{N}=4$. This result was better than MWPEr and MWPEt which produced the highest accuracy up to 93.94. The use of MWPE was better than the use of WPE at one decomposition level as in [8] which produced an accuracy of up to $70.71 \%$ at $\mathrm{N}=1 \mathrm{using}$ Bior 2.8. In the previous study, the use of WE as a feature only resulted in $43.43 \%$ accuracy using DWT Db2 level 7; while, the combination of six entropies yielded an accuracy of $94.95 \%$ [7].

In this study, all WPD subband results were used to calculate WPE. In another study, the subband selection of WPD results was performed to estimate the signal features. In [12], the information on each node became the basis for selecting the subband to be used as a feature. Meanwhile, in another paper, the distribution of data on the frequency spectrum was used as the basis for the gradual take up of subband [23]. The study of the best subband selection for
MWPE calculations will be a promising topic in subsequent research.

\section{CONCLUSION}

This paper describes the variation of MWPE calculations using Shannon entropy, Renyi entropy, and Tsallis entropy. Tests using five lung sound data classes showed that MPWE using Shannon entropy yielded higher accuracy compared to other two methods. This indicates that the distribution of energy in each subband is adequate to be the basis for the feature extraction of lung sound. MWPE is open for use in other biological sciences such as ECG, EEG, and heart sound.

\section{ACKNOWLEDGMENT}

This work has been financially supported by Ministry of Research, Technology, and Higher Education of Republic of Indonesia under Penelitian Disertasi Doktor Scheme no: 014/PNLT3/PPM/2018. 


\section{REFERENCES}

[1] Mondal, P. Bhattacharya, and G. Saha, "Detection of lungs status using morphological complexities of respiratory sounds.," Sci. World J., vol. 2014, pp. 1829-38, Jan. 2014.

[2] D. Sánchez Morillo, S. Astorga Moreno, M. Á. Fernández Granero, and A. León Jiménez, "Computerized analysis of respiratory sounds during COPD exacerbations.," Comput. Biol. Med., vol. 43, no. 7, pp. 914-21, Aug. 2013.

[3] A. Rizal, R. Hidayat, and H. A. Nugroho, "Pulmonary Crackle Feature Extraction using Tsallis Entropy for Automatic Lung Sound Classification," in The 1st 2016 International Conference on Biomedical Engineering (iBioMed), 2016, pp. 8-11.

[4] S. Charleston-Villalobos, L. Albuerne-Sanchez, R. Gonzalez-Camarena, M. Mejia-Avila, G. Carrillo-Rodriguez, and T. Aljama-Corrales, "Linear and Nonlinear Analysis of Base Lung Sound in Extrinsic Allergic Alveolitis Patients in Comparison to Healthy Subjects," Methods Inf. Med., vol. 52, no. 3, pp. 266-276, Apr. 2013.

[5] O. A. Rosso et al., "Wavelet entropy: a new tool for analysis of short duration brain electrical signals," J. Neurosci. Methods, vol. 105, pp. 65-75, 2001.

[6] F. Safara, S. Doraisamy, A. Azman, A. Jantan, and S. Ranga, "Wavelet packet entropy for heart murmurs classification," Adv. Bioinformatics, vol. 2012, 2012.

[7] A. Rizal, R. Hidayat, and H. A. Nugroho, "Entropy Measurement as Features Extraction in Automatic Lung Sound Classification," in the 3rd International Conference on Control, Electronics, Renewable Energy, and Communications 2017 (ICCEREC 2017), 2017.

[8] A. Rizal, R. Hidayat, and H. A. Nugroho, "Multilevel wavelet packet entropy: A new strategy for lung sound feature extraction based on wavelet entropy," in 2017 International Conference on Robotics, Automation and Sciences (ICORAS), 2017, pp. 1-5.

[9] A. Renyi, "On Measures of Entropy and Information," in Proceedings of the fourth Berkeley Symposium on Mathematics, Statistics and Probability, 1960, pp. 547-561.

[10] P. N. Rathie and S. Da Silva, "Shannon, Lévy, and Tsallis: A Note," Appl. Math. Sci., vol. 2, no. 28, pp. 1359-1363, 2008.

[11] P. Langley, "Wavelet Entropy as a Measure of Ventricular Beat Suppression from the Electrocardiogram in Atrial Fibrillation," Entropy, vol. 2015, pp. 6397-6411, 2015.
[12] F. Safara, S. Doraisamy, A. Azman, A. Jantan, and A. R. Abdullah Ramaiah, "Multi-level basis selection of wavelet packet decomposition tree for heart sound classification," Comput. Biol. Med., vol. 43, no. 10, pp. 1407-1414, 2013.

[13] R. Sharma and R. B. Pachori, "Classification of epileptic seizures in EEG signals based on phase space representation of intrinsic mode functions," Expert Syst. Appl., vol. 42, no. 3, pp. 1106-1117, 2015.

[14] J. Chen and G. Li, "Tsallis wavelet entropy and its application in power signal analysis," Entropy, vol. 16, no. 6, pp. 3009-3025, May 2014.

[15] M. Rosenblatt, A. Figliola, G. Paccosi, E. Serrano, and O. Rosso, "A Quantitative Analysis of an EEG Epileptic Record Based on MultiresolutionWavelet Coefficients," Entropy, vol. 16, pp. 5976-6005, 2014.

[16] X. Chen, D. Liu, G. Xu, K. Jiang, and L. Liang, "Application of wavelet packet entropy flow manifold learning in bearing factory inspection using the ultrasonic technique," Sensors (Switzerland), vol. 15, no. 1, pp. 341-351, 2015.

[17] C. Tsallis, "Possible generalization of Boltzman-Gibbs Statistics," $J$. Stat. Phys., vol. 52, no. 1/2, pp. 479-487, 1988.

[18] "The R.A.L.E Repository." [Online]. Available: http://www.rale.ca/Repository.htm. [Accessed: 22-Jul-2015].

[19] R. L. Wilkins, J. E. Hodgkin, and B. Lopez, Lung Sounds: A Practical Guide with Audio CD, 2nd ed. Maryland Heights, Missouri: Mosby, 1996.

[20] A. Rizal, R. Hidayat, and H. A. A. Nugroho, "Hjorth Descriptor Measurement on Multidistance Signal Level Difference for Lung Sound Classification," J. Telecommun. Electron. Comput. Eng., vol. 9, no. 2, pp. 23-27, 2017.

[21] A. Bohadana, G. Izbicki, and S. S. Kraman, "Fundamentals of lung auscultation.," N. Engl. J. Med., vol. 370, no. 8, pp. 744-51, Feb. 2014.

[22] A. Nakate and P. D. Bahirgonde, "Feature extraction of EEG signal using Wavelet Transform," Int. J. Comput. Appl., vol. 124, no. 2, pp. 21-24, 2015.

[23] A. Rizal, T. L. R. Mengko, and A. B. Suksmono, "Lung Sound Recognition Using Wavelet Packet Decomposition and ART2 ( Adaptive Resonance Theory 2 ) Neural Network," in Proceeding Biomedical Engineering Day 2006, 2006, vol. 2, pp. 2-6. 